\title{
Embryos for sale
}

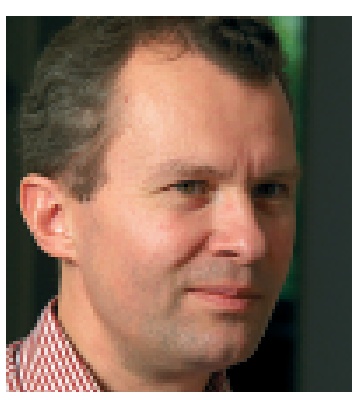

Christoph Rehmann-Sutter
A San Antonio, au Texas, il existe depuis peu une banque commerciale d'embryons. Avant de passer commande, on peut recueillir des informations sur la race, le niveau d'études, l'apparence et les caractéristiques personnelles des donneurs de semence et d'ovule à qui il est fait appel pour la fécondation. Le quotidien Washington Post en a parlé le 6 janvier dernier et la NZZ le dimanche 14 janvier. Le fournisseur est l'Abraham Center of Life (quel nom!), qui organise ou procure aussi des enfants adoptifs, des dons d'ovule, des mères d'adoption et des embryons surnuméraires issus de la fécondation in vitro (FIV).

L'avantage certain de la banque d'embryons se situe dans les coûts. Avec 2500 dollars par embryon, une grossesse peut coûter moins de 10000 dollars, ce qui est meilleur marché qu'une adoption régulière ou une FIV. Sur le plan de la production, proposer directement des embryons va de soi si l'on offre de toute façon des ovules et des spermatozoïdes aux qualités testées. Aux yeux du client, il ne s'agit de rien d'autre que d'une simple combinaison d'un ovule et d'un éjaculat, «confectionnés» déjà en embryon. Et le Centre produit en série: un nouveau pas qui peut sembler petit lui aussi. Une donneuse d'ovule et un donneur de semence ont permis de fabriquer un lot de 22 embryons. Deux clientes, une en Californie, l'autre au Canada, enceintes actuellement de 6 mois, se partagent cette série. Ce sont des chiffres bas, mais il s'agit de voir la logique industrielle qui en ressort.

Il convient de relever le motif donné à ses clientes par la directrice du Centre dans une lettre (le document figure sur la page internet du centre): tous les pays industrialisés, dit-elle, sont touchés par une crise à long terme, le «collapsus de la fécondité». Le taux de fécondité des Américains n'est plus élevé que celui du Japon et de l'Allemagne que parce qu'aux Etats-Unis, les immigrants hispaniques affichent un taux de 2,9. Mme Ryan évite de donner une motivation directement eugénique, elle met plutôt l'accent sur la situation de ses clientes et clients. Les couples les surnuméraires de la FIV, parce qu'ils provien- nent d'ovules et de spermatozoïdes de donneurs connus. En plus, il y a moins de complications émotionnelles que dans les familles qui placent un embryon surnuméraire dans une autre famille.

En éthique et en biopolitique, une polémique règne depuis des années sur l'autonomie reproductive. Or, si l'on interprète cette notion comme étant liée à la conception en vigueur de l'autonomie du client sur le libre marché (rapport prix-efficacité en relation avec la capacité financière), cela affecte la relation que nous avons avec l'enfant. Les partisans de l'autonomie reproductive argumentent que nous ne limitons pas non plus la reproduction chez les personnes qui peuvent avoir des enfants sans aide médicale. $\mathrm{Vu}$ sous cet angle, on ne saurait s'opposer à l'élargissement du principe du marché et de l'industrie à la reproduction assistée, tant qu'il n'y a pas de dommage et aussi longtemps qu'elle répond au souhait des patientes.

L'idée de l'autonomie reproductive est issue du débat sur la contraception et l'interruption de grossesse. Il s'agit là du sujet central du droit de la femme à n'avoir un enfant que si elle le désire. L'autonomie reproductive est donc un droit de défense. Les nouvelles techniques de reproduction, quant à elles, permettent en revanche à des couples de devenir parents, ce qui représente un engagement. Avoir des enfants implique une longue relation porteuse de responsabilité. Une relation qui se situe hors des règles du jeu du marché. Un principe motivé par un droit de défense contre les grossesses non désirées ne peut pas s'appliquer sans autre aux techniques de reproduction.

Cet argument ne saurait signifier le rejet des techniques de reproduction, il incite plutôt à faire preuve de prudence envers les mécanismes de l'économie de marché. Il nous montre qu'il est justifié de considérer d'autres aspects de la question: il faut des règles qui en priorité doivent viser le bien de l'enfant. Y a-t-on suffisamment pensé lorsqu'on a rationalisé les conditions de la production en fonction des conditions du marché, à courte vue? Il se peut que l'enfant veuille savoir comment il a été engendré, qu'il veuille pouvoir s'identifier positivement à ses origines. Est-ce aussi prévu?

Christoph Rehmann-Sutter* qui ne peuvent avoir d'enfants ont de plus en plus de difficultés à adopter. Une banque d'embryons peut apporter une aide certaine. Par ce biais, les clients peuvent notamment prétendre recevoir des embryons de meilleure qualité que de rédaction du BMS pour les questions d'éthique. 